\title{
The penetration of meridional flow into the tachocline and its meaning for the solar dynamo
}

\author{
G. Rüdiger ${ }^{1}$, L. L. Kitchatinov ${ }^{1,2}$, and R. Arlt ${ }^{1}$ \\ 1 Astrophysikalisches Institut Potsdam, An der Sternwarte 16, 14482 Potsdam, Germany \\ e-mail: [gruediger; rarlt]@aip.de \\ 2 Institute for Solar-Terrestrial Physics, PO Box 4026, Irkutsk 664033, Russian Federation \\ e-mail: kit@iszf.irk.ru
}

Received 26 August 2005 / Accepted 26 October 2005

\section{ABSTRACT}

The penetration of meridional flow into the radiative solar interior is computed with a mean-field model for global circulation in convective zones. The results clearly show that the penetration under solar conditions belongs to the Ekman regime. The penetration is too weak to play any role in the solar dynamo mechanism. We argue, however, that this penetration is sufficient to configure the internal magnetic field of the radiative core so that the solar tachocline can be explained as a Hartmann layer. The resulting meridional flow at the base of the convection zone is fast enough $(\sim 7 \mathrm{~m} / \mathrm{s})$ to realize an advection-dominated $\alpha \Omega$-dynamo within the convection zone.

Key words. hydrodynamics - Sun: interior - stars: interiors

\section{Motivation}

The penetration of the meridional flow from the solar convection zone into the radiative core beneath the convection zone is currently debated in the context of dynamo models for solar activity (Küker et al. 2001; Nandy \& Choudhuri 2002; Chatterjee et al. 2004; Gilman \& Miesch 2004; Dikpati et al. 2005). The meridional circulation is a principal ingredient of advectiondominated dynamo models which are quite successful in reproducing the observed parameters of the 11-year solar cycle (Choudhuri et al. 1995; Dikpati \& Gilman 2001; Bonanno et al. 2002). Such a dynamo is called "advection-dominated" if the time $\left(\tau_{\text {adv }}\right)$ of the magnetic field advection by the meridional flow $u^{\mathrm{m}}$ is much shorter than the diffusion time $\left(\tau_{\text {diff }}\right)$ of the field, i.e. if

$\widetilde{\mathrm{Rm}}=\frac{\tau_{\text {diff }}}{\tau_{\mathrm{adv}}} \gg 1$.

The time scales of the penetration region can be estimated as follows: $\tau_{\text {adv }} \simeq R_{\text {in }} / u^{\mathrm{m}}, \tau_{\text {diff }} \simeq D_{\text {pen }}^{2} / \eta_{\text {in }}$ with $D_{\text {pen }}$ as the penetration depth of the meridional flow into the radiative zone ( $R_{\text {in }}$ is the radius of the base of the convection zone and $\eta_{\text {in }}$ is the magnetic diffusivity beneath $R_{\text {in }}$ ). The modified magnetic Reynolds number (1) then becomes

$\widetilde{\mathrm{Rm}}=\left(\frac{D_{\text {pen }}}{R_{\text {in }}}\right)^{2} \mathrm{Rm}$,

with the ordinary magnetic Reynolds number $\mathrm{Rm}=R_{\mathrm{in}} u^{\mathrm{m}} / \eta_{\text {in }}$ of the meridional flow. For $u^{\mathrm{m}}$ of about $2 \mathrm{~m} / \mathrm{s}$ the magnetic
Reynolds number Rm is about $10^{13} \mathrm{~cm}^{2} \mathrm{~s}^{-1} / \eta_{\text {in }}$. It is thus a very large number for microscopic values of $\eta_{\text {in }}$ but it is only of order 1-10 for turbulent values. We shall show that indeed microscopic $\eta$-values should be relevant to the region below the solar convection zone but in this case the penetration depth becomes so small that $\widetilde{\mathrm{Rm}}$ remains a small number - and the penetration looses any relevance for advection-type dynamos.

This Letter presents the results of numerical simulations for the meridional flow in the solar convection zone and its penetration into the layer beneath the convection zone. The simulations are performed with the model of global circulation inside the stellar convection envelopes (Kitchatinov \& Rüdiger 1999) which has been modified to include penetration. The results suggest that a meridional flow sufficient for advectiondominated dynamo exists at the base of the convection zone. Its penetration into the layer beneath the convection zone strongly depends on the viscosity there. It is further shown that the rotation law beneath the convection zone (in the "tachocline") is hydrodynamically stable so that the viscosity should be very small there. If this is true, then the penetration effect should be much too weak for being important for the dynamo mechanism.

\section{The model}

The flow pattern inside the star is simulated by solving numerically the mean-field momentum equation

$(\overline{\boldsymbol{u}} \cdot \nabla) \overline{\boldsymbol{u}}+\frac{1}{\rho} \nabla \cdot(\rho Q)+\frac{1}{\rho} \nabla P=\boldsymbol{g}$ 
for the axisymmetric mean velocity $\boldsymbol{u}^{\mathrm{m}}$. Here $Q$ is the correlation tensor of the fluctuating velocities,

$Q_{i j}=\left\langle u_{i}^{\prime}(\boldsymbol{x}, t) u_{j}^{\prime}(\boldsymbol{x}, t)\right\rangle$,

which includes the eddy viscosity as well as the nondiffusive terms representing the $\Lambda$-effect. Equation (3) is solved together with the entropy equation

$\rho T \boldsymbol{u}^{\mathrm{m}} \cdot \nabla S+\operatorname{div}\left(\boldsymbol{F}^{\mathrm{conv}}+\boldsymbol{F}^{\mathrm{rad}}\right)=0$.

The specific entropy $S$ is needed to define the baroclinic meridional flow as well as the eddy transport coefficients for the nonrotating case, e.g., the eddy viscosity

$v_{\mathrm{T}}=-\frac{\tau_{\text {corr }} \ell_{\text {corr }}^{2} g}{15 c_{\mathrm{p}}} \frac{\partial S}{\partial r}$.

For positive $\partial S / \partial r$ the eddy viscosity vanishes.

The equations are solved in a spherical layer inside a star. The external boundary $\left(R_{\text {out }}\right)$ is placed shortly below the photosphere to exclude the outermost layer with its extremely strong density stratification $\left(R_{\text {out }} / R_{\odot}=0.96\right)$. Both the stress-free and no-penetration $\left(\bar{u}_{r}=0\right)$ boundary conditions are there applied. The thermal conditions assume constant and spherically symmetric heat flux at the bottom and free radiation on the top of the domain. The model design is very close to that of Kitchatinov \& Rüdiger (1999) with two differences. A slightly modified $\Lambda$-effect is used for the upper layers which, however, does not influence the meridional flow penetration (see Kitchatinov \& Rüdiger 2005).

The other modification is more general. The lower boundary is now placed deeper inside the radiative zone to allow the penetration. The diffusivities there are free parameters, called $\nu_{\text {in }}$ and $\chi_{\text {in }}$ for the viscosity and the thermal diffusivity, resp. The real values of the diffusivities depend on the stability of the tachocline. The numerical code has a sufficient resolution (about 300 grid points in the penetration region) to resolve even a shallow penetration but, of course, it cannot work with the true microscopic viscosity $\left(v_{\mathrm{in}} \simeq 10 \mathrm{~cm}^{2} \mathrm{~s}^{-1}\right)$ of the core. The dependence of the penetration depth on a given $v_{\text {in }}$ and other parameters is computed.

\section{Stability of the tachocline}

Whether the viscosity $v_{\text {in }}$ is large or small beneath the convection zone depends strongly on the stability of the solar tachocline. If the latter is unstable then the viscosity is large and the penetration can be expected as deep. Is it, however, stable then the viscosity is small and the penetration may be very weak. If the solar tachocline is considered as a shear flow then it should be unstable for sufficiently high Reynolds numbers of rotation and sufficiently high equator-pole differences of the angular velocity at its upper boundary.

The hydrodynamical stability/instability of the solar tachocline has been probed with the Hollerbach code (Hollerbach 2000) for a thin spherical shell subject to differential rotation (Arlt et al. 2005). If the shear is formed only by latitudinal gradients of $\Omega$ (the case considered by Watson 1981, and also Charbonneau et al. 1999) then the onset of the (linear)

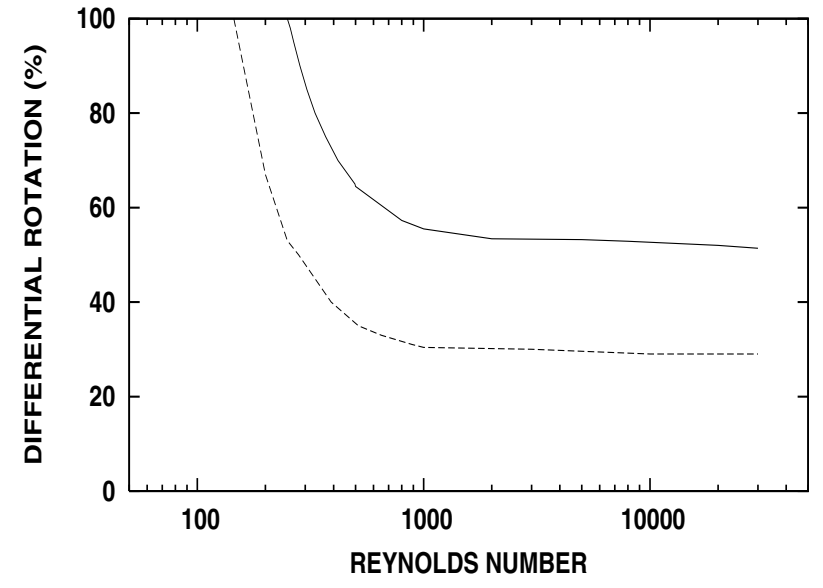

Fig. 1. Bifurcation map for the hydrodynamic shear instability of the solar tachocline. Dashed: only latitudinal shear, solid: the real rotation law $\Omega=\Omega(r, \theta)$ taken from helioseismology. Note that the solar tachocline is not unstable in the hydrodynamical regime.

instability starts at about ${ }^{1} 29 \%$ equator-pole difference of $\Omega$. If, however, the equator-pole difference decreases inwards - as it is true for the $\Omega$-profile in the solar tachocline - then a much stronger latitudinal shear is necessary for the onset of the instability (Fig. 1). Rotation laws with (say) 30\% equator-pole difference at the upper boundary are always stable for the given Reynolds numbers and Rayleigh numbers.

The importance of the three-dimensional treatment of the stability problem is based on the coupling between infinitesimal shells each having their individual latitudinal shear. The coupling can be caused by both radial flows and viscosity. Figure 1 indicates a very weak dependence of the marginal stability line on the Reynolds number, i.e. on the viscosity. Nevertheless, a remaining even though very small viscosity is separating significantly the stability of the three-dimensional from the two-dimensional computations. Computations in which the radial flow is cancelled artificially show that the stability limit changes only by about $1 \%$. We therefore assume that even at a small microscopic viscosity the radial dependence of the shear makes the tachocline more stable than a purely latitudinal one.

As a consequence, the effective viscosity necessary for the penetration flow should be very small because of the lack of an instability which could provide (anisotropic) eddy viscosities. For final statements about this problem the negative buoyancy (strongly negative Rayleigh numbers in the Boussinesq approximation), nonlinear effects (higher Reynolds numbers) as well as the consequences of strong toroidal magnetic fields must still be taken into account (see Gilman \& Fox 1997; Brandenburg $\&$ Schmitt 1998; Sule et al. 2005).

Rüdiger \& Kitchatinov (1997) have shown that an Hartmann layer approximation of the solar tachocline leads to the estimate

$$
\frac{D_{\mathrm{tach}}}{D} \simeq \mathrm{Ha}^{-0.5}
$$

\footnotetext{
${ }^{1}$ Slightly depending on the form of the latitudinal profile.
} 

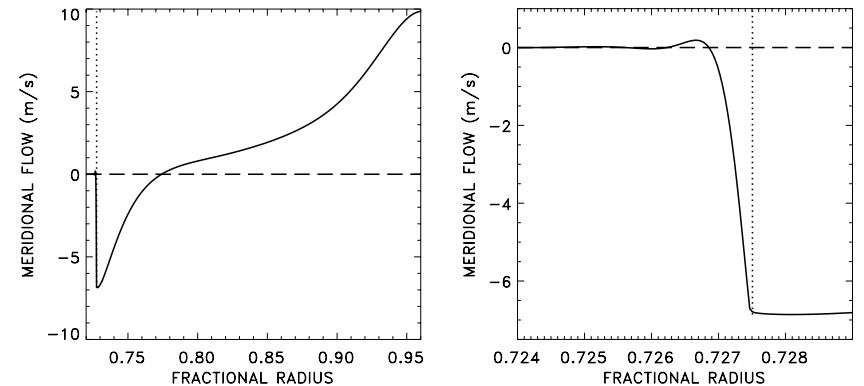

Fig. 2. Meridional velocity at $45^{\circ}$ latitude as function of radius for the entire simulation domain (left) and for the penetration region (right). Positive velocity means poleward flow. The vertical dotted line shows the position of the bottom of the convection zone. Here $v_{\text {in }}=1.1 \times$ $10^{9} \mathrm{~cm}^{2} \mathrm{~s}^{-1}$.

for the ratio of the magnetic $\left(D_{\text {tach }}\right)$ to the nonmagnetic $(D)$ tachocline thickness. Without magnetic fields, the differential rotation imposed by the convection zone will thread the entire radiative core. One can therefore use $D \simeq R_{\text {in }}$. The Hartmann number Ha of the given poloidal field then is

$\mathrm{Ha}=\frac{R_{\text {in }} \cdot B}{\sqrt{\mu_{0} \rho v_{\text {in }} \eta_{\text {in }}}} \cdot$

We find from Eqs. (7) and (8)

$\frac{D_{\text {tach }}}{R_{\text {in }}} \simeq 10^{-5}\left(\frac{\nu_{\text {in }} \eta_{\text {in }}}{B^{2}}\right)^{0.25}$

(in c.g.s.) so that for (say) $v_{\text {in }} \simeq 10 \mathrm{~cm}^{2} \mathrm{~s}^{-1}$ and (say) $\eta_{\text {in }} \simeq$ $10^{3} \mathrm{~cm}^{2} \mathrm{~s}^{-1}$ a field of only $10^{-4} \mathrm{G}$ is enough to produce the very thin solar tachocline. The resulting toroidal field $B_{\phi}$ does not depend on the initial poloidal field $B$ and becomes

$V_{\mathrm{A}} \simeq \sqrt{\frac{\nu_{\mathrm{in}}}{\eta_{\text {in }}}} U_{0}=\sqrt{\operatorname{Pm}} U_{0}$,

(as an order of magnitude), with $V_{\mathrm{A}}=B_{\phi} / \sqrt{\mu_{0} \rho}$ as the Alfvén velocity and $U_{0}=R_{\mathrm{in}} \Omega$ the linear velocity of the rotation. One finds $B_{\phi} \simeq 10^{3 \ldots 4} \mathrm{G}$ for the Sun.

If turbulent values for the dissipation are used, the toroidal fields result in $10^{4 . .5} \mathrm{G}$ but the poloidal field $B$ required to form a tachocline according to Eq. (9) is then by far too high $\left(10^{6} \mathrm{G}\right)$. The reason is that the toroidal field is fixed by the ratio of both the diffusivities while the poloidal field is fixed by their product.

\section{Results and discussion}

In all our calculations the meridional flow in the convection zone forms a single cell with poleward motion at the top and equatorward motion at the base of the convection zone. This is a very robust result. The circulation beneath the convection zone is much more complicated. Figure 2 (right) shows several reversals of the meridional velocity with depth in this region. The velocity amplitude decreases after each reversal. The penetration depth $\left(D_{\text {pen }}\right)$ can thus be defined as the distance from the base of the convection zone to the location of first reversal of $\bar{u}_{\theta}$.

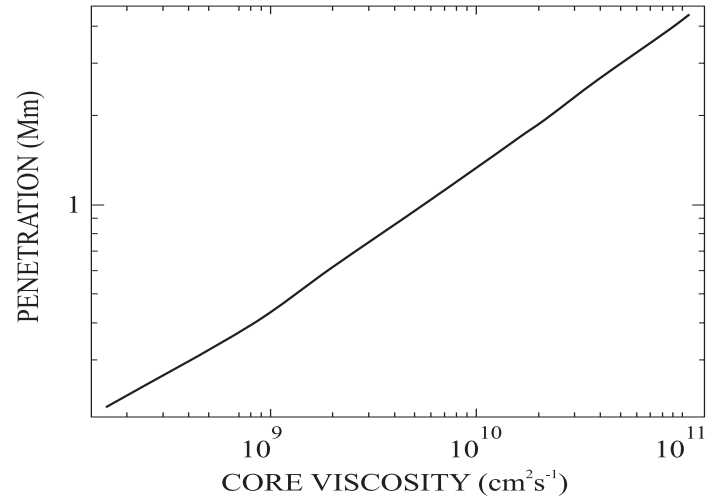

Fig. 3. Penetration depth $D_{\text {pen }}$ of the meridional flow into the radiative interior at latitude $45^{\circ}$ in dependence on the viscosity $v_{\text {in }}$ prescribed for the radiation interiour. The penetration depth runs with $v_{\mathrm{in}}^{0.5}$.

Note that for a viscosity of $10^{10} \mathrm{~cm}^{2} \mathrm{~s}^{-1}$ the penetration depth is (only) $1000 \mathrm{~km}$ (Fig. 3). Figure 3 yields a power law

$D_{\text {pen }} \sim v_{\text {in }}^{0.5}$

between the penetration depth and the given inner viscosity $v_{\text {in }}$. This numerical result complies with the conclusion of Gilman $\&$ Miesch (2004) that under solar conditions the penetration belongs to the Ekman regime. Penetration of this type results from the viscous drag imposed by the meridional circulation at the base of the convection zone on the fluid beneath. The relation (11) also agrees with the standard estimation of the Ekman depth,

$D_{\text {pen }} \simeq \sqrt{\frac{\nu_{\text {in }}}{2 \Omega}}$.

This interpretation is supported by the numerical finding that any variation of the thermal conductivity for one and the same $v_{\text {in }}$ does not influence the penetration depth.

The inclusion of the (stably stratified) tachocline into the calculations also affects the global circulation and thermodynamics within the convection zone. The meridional flow at the base of the convection zone is reduced and the equator-to-pole temperature difference is increased. This is in full agreement with the results of Rempel (2005) who found that the equilibrium of forces in the tachocline region demands a latitudinal entropy gradient which is transmitted into the convection zone by radial diffusion. The poles become warmer by this effect and the equator-pole temperature difference increases beyond the value resulting from anisotropic heat-diffusion inside the convection zone. We find, however, that the influence of the tachocline on the rotation law of the convection zone reduces with decreasing $v_{\text {in }}$ and $\chi_{\text {in }}$ (here always the Prandtl number $\operatorname{Pr}=v_{\text {in }} / \chi_{\text {in }}$ is $\left.1 / 8\right)$. For the smallest $v_{\text {in }}$ in Fig. 3 the effect of the tachocline almost disappears. The rotation law given in Fig. 4 obtained with $v_{\text {in }} \simeq 1.1 \times 10^{9} \mathrm{~cm}^{2} \mathrm{~s}^{-1}$ is almost the same as in models without penetration.

The importance of the penetration effect for the dynamo mechanism can be estimated by the ratio $\widetilde{\mathrm{Rm}}$ of the advection time and the diffusion time. With Eq. (12) the ratio (1) becomes

$\widetilde{\mathrm{Rm}} \propto \operatorname{Pm} \frac{u^{\mathrm{m}}}{R_{\mathrm{in}} \Omega}$ 


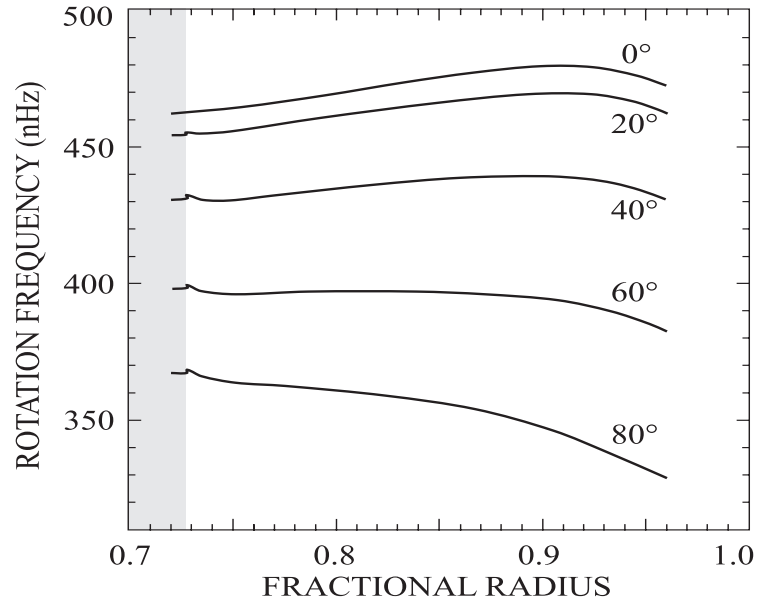

Fig. 4. Rotation law for the same model used in Fig. 2. The rotation law for the small $v_{\text {in }}\left(\sim 10^{9} \mathrm{~cm}^{2} \mathrm{~s}^{-1}\right)$ is very close to the model without penetration. The numbers mark the latitude.

which is very small unless for the magnetic Prandtl number, Pm, an unrealistically huge value is adopted ${ }^{2}$. With other words, the penetration layer is so thin in the Ekman regime that any induced magnetic field immediately diffuses away and the meridional flow cannot transport it to the equator. We have to conclude that the penetration of the meridional flow into the radiative zone is not significant for magnetic field advection. This statement remains true even for as turbulent tachocline as long as the turbulent Prandtl number remains of order unity or smaller (Yousef et al. 2003)

The penetration, however, is important for the Hartmann layer models of the solar tachocline which work with a weak internal magnetic field in the radiative interiour. Those models are only successful if the radial component, $B_{r}$, of the internal field in the tachocline region is small compared to its meridional component, $B_{\theta}$ (MacGregor \& Charbonneau 1999). The penetration, indeed, leads to such a magnetic geometry if

$\frac{B_{r}}{B_{\theta}} \sim \frac{\eta_{\text {in }}}{u^{\mathrm{m}} D_{\text {pen }}} \ll 1$.

One finds with Eq. (12) the condition

$\left(u^{\mathrm{m}}\right)^{2} \gg \frac{\eta_{\mathrm{in}}^{2} \Omega}{v_{\mathrm{in}}}$

or - using the Reynolds number

$\operatorname{Re}=\frac{u^{\mathrm{m}} R_{\text {in }}}{v_{\text {in }}}$

of the meridional flow - the final formulation is

$\operatorname{Re} \gg \frac{R_{\text {in }} \Omega}{u^{\mathrm{m}}} \mathrm{Pm}^{-2}$.

It can never be fulfilled with the high values of the eddy viscosity $\left(v_{\text {in }}>10^{7} \mathrm{~cm}^{2} \mathrm{~s}^{-1}\right)$, so that indeed the Hartmann layer concept of the tachocline requires the existence of high Reynolds numbers for the penetrating meridional flow, i.e. it requires

${ }^{2}$ It is $\mathrm{Pm} \sim 10^{-2}$ for microscopic diffusivities and $\mathrm{Pm} \lesssim 1$ for turbulent diffusivities. small values of the viscosity excluding the "fast" tachocline models.

The amplitude of the meridional flow of Fig. 2 at the base of the convection zone $(\sim 7 \mathrm{~m} / \mathrm{s})$ is fast enough to realize an advection-dominated dynamo inside the convection zone. The characteristic advection time from pole to equator with this velocity is about 4 years.

\section{Conclusions}

Our argumentation is as follows. After relation (13) the penetration depth is always so thin that it cannot play an important role for the advection-dominated dynamo. This is in particular true if the layer beneath the convection zone (the "tachocline") has a small magnetic Prandtl number which belongs to any nonturbulent stratification.

At this point we therefore emphasize the plausibility of a nonturbulent tachocline. First there is the hydrodynamical stability of the rotation profile in the tachocline as was shown earlier. Second the magnetic formation of the tachocline is only possible for small diffusivities. Otherwise the poloidal fields required would be unreasonably large. The likely stability of the tachocline thus favours extremely small penetration depths. We have to add though that the magnetohydrodynamical stability or instability of the tachocline in a fully three-dimensional treatment is still an open but important question.

Acknowledgements. L.L.K. is grateful to the Alexander v. Humboldt Foundation and to the Astrophysical Institute Potsdam for hospitality and the visitor support. The Russian Foundation for Basic Research is also acknowledged (project 05-02-16326).

\section{References}

Arlt, R., Sule, A., \& Rüdiger, G. 2005, A\&A, 441, 1171

Bonanno, A., Elstner, D., Rüdiger, G., \& Belvedere, G. 2002, A\&A, 390,673

Brandenburg, A., \& Schmitt, D. 1998, A\&A, 338, L55

Charbonneau, P., Dikpati, M., \& Gilman, P. A. 1999, ApJ, 526, 523

Chatterjee, P., Nandy, D., \& Choudhuri, A. R. 2004, A\&A, 427, 1019

Choudhuri, A. R., Schüssler, M., \& Dikpati, M. 1995, A\&A, 303, L29

Dikpati, M., \& Gilman, P. A. 2001, ApJ, 559, 428

Dikpati, M., Rempel, M., Gilman, P. A., \& MacGregor, K. B. 2005, A\&A, 437, 699

Gilman, P. A., \& Fox, P. 1997, ApJ, 484, 439

Gilman, P. A., \& Miesch, M. S. 2004, ApJ, 611, 568

Hollerbach, R. 2000, Int. J. Numer. Meth. Fluids, 32, 773

Kitchatinov, L. L., \& Rüdiger, G. 1999, A\&A, 344, 911

Kitchatinov, L. L., \& Rüdiger, G. 2005, Astron. Nachr., 326, 379

Küker, M., Rüdiger, G., \& Schultz, M. 2001, A\&A, 374, 301

MacGregor, K. B., \& Charbonneau, P. 1999, ApJ, 519, 911

Nandy, D., \& Choudhuri, A. R. 2002, Science, 296, 1671

Rempel, M. 2005, ApJ, 622, 1320

Rüdiger, G., \& Kitchatinov, L. L. 1997, Astron. Nachr., 318, 273

Sule, A., Rüdiger, G., \& Arlt, R. 2005, A\&A, 437, 1061

Watson, M. 1981, Geophys. Astrophys. Fluid Dyn., 16, 285

Yousef, T., Brandenburg, A., \& Rüdiger, G. 2003, A\&A, 411, 321 